\title{
An Unusual Complication of Cabergoline Treatment of Macroprolactinomas
}

\author{
Amal Moumen ${ }^{\mathrm{a}, \mathrm{e}}$, Sanae Chakdoufia ${ }^{\mathrm{a}}$, Hamza El Jadi ${ }^{\mathrm{b}}$, Ouijdane Zammanic, \\ Cherif Abad El Asrid, Ghizlaine Belmejdoub ${ }^{\mathrm{a}}$
}

\begin{abstract}
Dopamine agonist (DA) treatment is the first choice treatment of prolactinomas. Optic chiasm prolapse with secondary visual deterioration is an unusual and rare complication of DA treatment. A 40-year-old man with giant prolactinoma was successfully treated by cabergoline. After 14 months, he presented with a visual impairment with no ophthalmologic anomalies that could explain this worsening. The pituitary magnetic resonance imaging (MRI) revealed an important shrinkage of prolactinoma with a prolapse and a central atrophy of optic chiasm secondary to the adenoma shrinkage. This case highlights the need of regular assessment of visual field all along cabergoline treatment of macroprolactinomas despite initial improvement or even normalization of visual field, to promptly identify an optic chiasm prolapse and avoid the optic chiasm atrophy.
\end{abstract}

Keywords: Cabergoline; Optic chiasm prolapse; Macroprolactinoma

\section{Introduction}

Prolactinomas represent $30-40 \%$ of all pituitary tumors [1]. Dopamine agonist (DA) is the first-line treatment of prolactinomas even giant macroprolactinomas with visual deterioration. Cabergoline has been reported as the most effective in normalizing prolactin levels and inducing tumor shrinkage [2]. Common side effects of DA treatment include headaches, nau-

Manuscript submitted February 19, 2021, accepted March 9, 2021

Published online April 25, 2021

${ }^{a}$ Endocrinology and Diabetology Department, Mohammed V Military Hospital, Faculty of Medicine and Pharmacy, Mohammed V University, Rabat, Morocco

bEndocrinology and Diabetology Department, Avicenne Military Hospital, Marrakech, Morocco

'Radiology Department, Mohammed V Military Hospital, Faculty of Medicine and Pharmacy, Mohammed V University, Rabat, Morocco

${ }^{\mathrm{d} N e u r o s u r g e r y}$ Department, Mohammed V Military Hospital, Faculty of Medicine and Pharmacy, Mohammed V University, Rabat, Morocco

${ }^{\mathrm{e}}$ Corresponding Author: Amal Moumen, Endocrinology and Diabetology Department, Mohammed V Military Hospital, Avenue des FAR-Hay-Riad, CP 10100, Rabat, Morocco. Email: moumen2105@gmail.com

doi: https://doi.org/10.14740/jem730 sea and vomiting, fatigue, orthostatic hypotension, peripheral vascular changes and depression [3]. Pituitary apoplexy and cerebrospinal fluid rhinorrhea are considered rare side effects of DA treatment $[4,5]$. We report an unusual case of secondary visual field deterioration during cabergoline treatment of a giant prolactinoma, despite tumor shrinkage, related to optic chiasm prolapse.

\section{Case Report}

A 40-year-old man with no medical history presented with chronic headaches, progressive visual field loss, poor libido and erectile dysfunction. The patient also reported a long history of tiredness, cold intolerance, constipation and gain weight without lifestyle changes. General examination revealed a bradycardia with a pulse rate at $54 / \mathrm{min}$, blood pressure at $100 / 60 \mathrm{~mm} \mathrm{Hg}$ and a body mass index of $32 \mathrm{~kg} / \mathrm{m}^{2}$. He had a pale face with thinning of the outer third of the eyebrow and bilateral gynecomastia. Ophthalmologic exam showed a visual acuity of $4 / 10$ at the right eye and 1/10 at the left eye. Visual field perimetry revealed a right superior temporal quadrantanopsia and left temporal hemianopsia (Fig. 1a). Pituitary magnetic resonance imaging $(\mathrm{MRI})$ revealed a heterogeneous giant pituitary adenoma of $50 \times 47 \times 27 \mathrm{~mm}$ in diameter, moderately enhanced following gadolinium injection, bulging in the sphenoidal sinus and with bilateral contact with cavernous sinuses. The adenoma was extending into the suprasellar region, thus compressing the optic chiasm.

Formal endocrine testing revealed panhypopituitarism and a markedly elevated prolactin level of $>10,000 \mathrm{ng} / \mathrm{mL}$ (normal range $<25 \mathrm{ng} / \mathrm{mL}$ ), confirming the diagnosis of macroprolactinomas. Insulin-like growth factor-1 (IGF-1) was normal.

The patient was started on cabergoline treatment $2 \mathrm{mg} /$ week in addition to replacement therapy initially with hydrocortisone (20 mg daily) and then with L-thyroxine (50 $\mu \mathrm{g}$ daily). After 1 month of cabergoline, patient reported a subjective visual improvement but prolactin level was still elevated at $>$ $470 \mathrm{ng} / \mathrm{mL}$ and visual field perimetry was worsening. The cabergoline doses were increased to $3 \mathrm{mg} /$ week with subsequent normalization of prolactin level and visual acuity and visual field improvement (Fig. 1b). Three months later, pituitary MRI showed a decrease of the adenoma size to $28 \times 33 \times 29 \mathrm{~mm}$ without contact with the optic chiasm (Fig. 2).

After 14 months of cabergoline treatment, the ophthal- 


\section{Left eve}
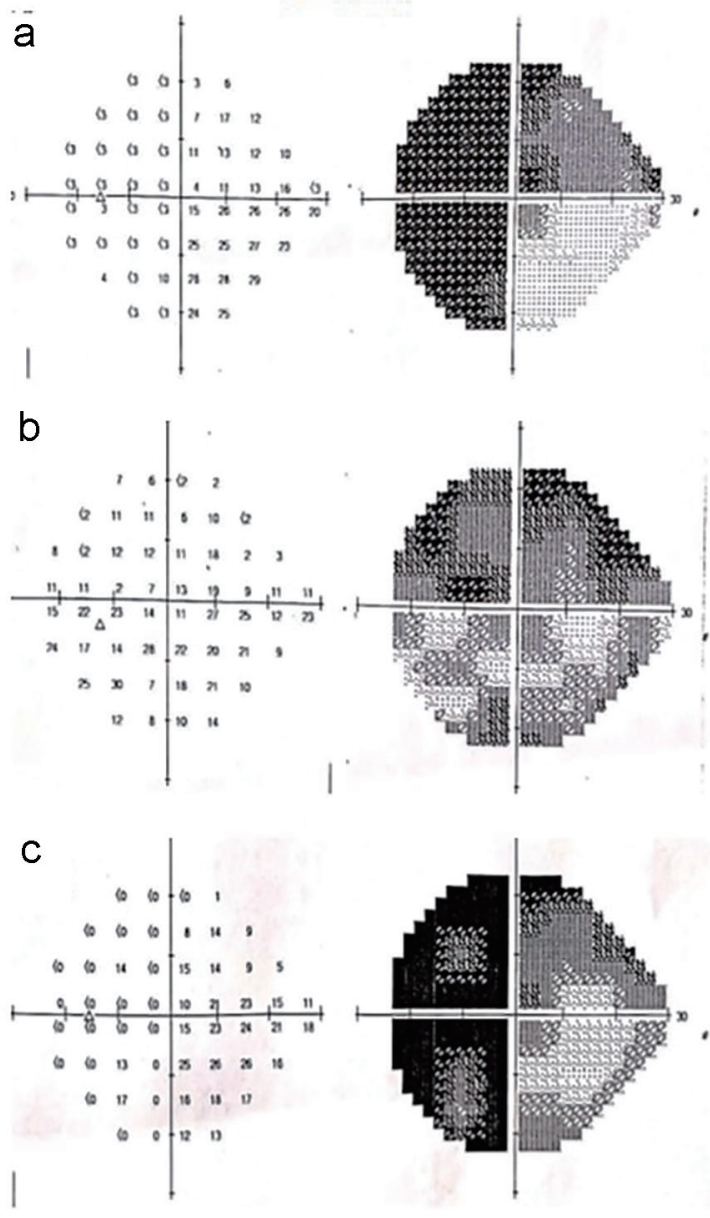

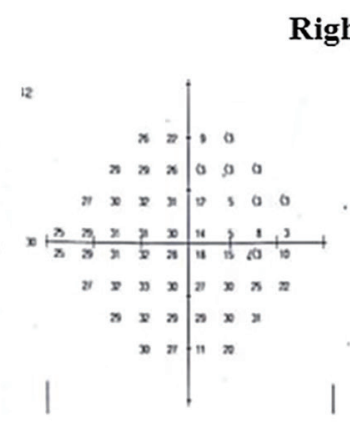

Right eve
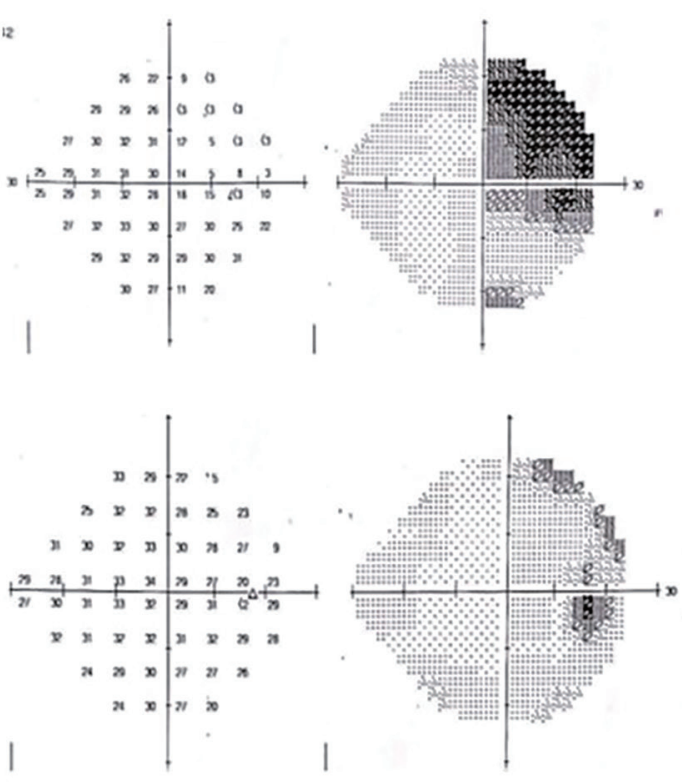

Figure 1. (a) Initial visual fields showing right superior temporal quadrantanopsia and left temporal hemianopsia. (b) After 2 months of cabergoline treatment, visual fields showing improvement in the temporal fields. (c) After 14 months of cabergoline treatment, visual field showing a secondary deterioration of temporal field.

mologist reported a secondary visual field deterioration (Fig. 1c) with no ophthalmologic anomalies that could explain this worsening. An urgent pituitary MRI was conducted to exclude pituitary hemorrhage or infarction. It revealed an important shrinkage of adenoma's height from 28 to $6 \mathrm{~mm}$ with a prolapse and central atrophy of optic chiasm secondary to tumor shrinkage (Fig. 3).

Cabergoline treatment was withdrawn for 2 weeks, and then restarted at a lower dose (4 mg/week) with stabilization of visual fields and visual acuity.

\section{Discussion}

Secondary deterioration in visual fields after successful medical treatment for a macroprolactinoma may be caused by pituitary apoplexy or tumor regrowth. Optic chiasm prolapse secondary to dramatic shrinkage of macroprolactinoma is a rare and an under-recognized complication of DA treatment [6]. The optic chiasm herniation has been described in patients with macroprolactinoma, treated with bromocriptine [6-8], pergolide [9], or cabergoline [10]. The mechanism of the secondary visual impairment due to optic chiasm prolapse after DA treatment is unclear. The adherence of the adenoma to the suprasellar visual system before DA treatment could predispose to the herniation upon reduction of the adenoma size by treatment and subsequent development of empty sella $[6,7$, 11]. The chiasm or optic nerves were dragged down into the sella by scarred tissue and adherence may directly compromise the chiasm's vascular supply $[6,7]$. However, prolapse of the optic chiasm without visual field defect is very common and no correlation has been found between the degree of chiasmal herniation and the severity of the visual loss [7]. Direct toxicity of dopaminergic agonist agents, vasospasm-induced ischemia, or reversible perivascular fibrosis has been suggested [10]. This is consistent with the improvement in visual fields observed after withdrawal or reduction in therapeutic dosage [10].

In our case, the tumor was initially adherent to the suprasellar visual system and the increase of cabergoline dose may have induced intratumoral fibrosis, tumor shrinkage and alteration of vascular supply, thus causing optic chiasm prolapse 

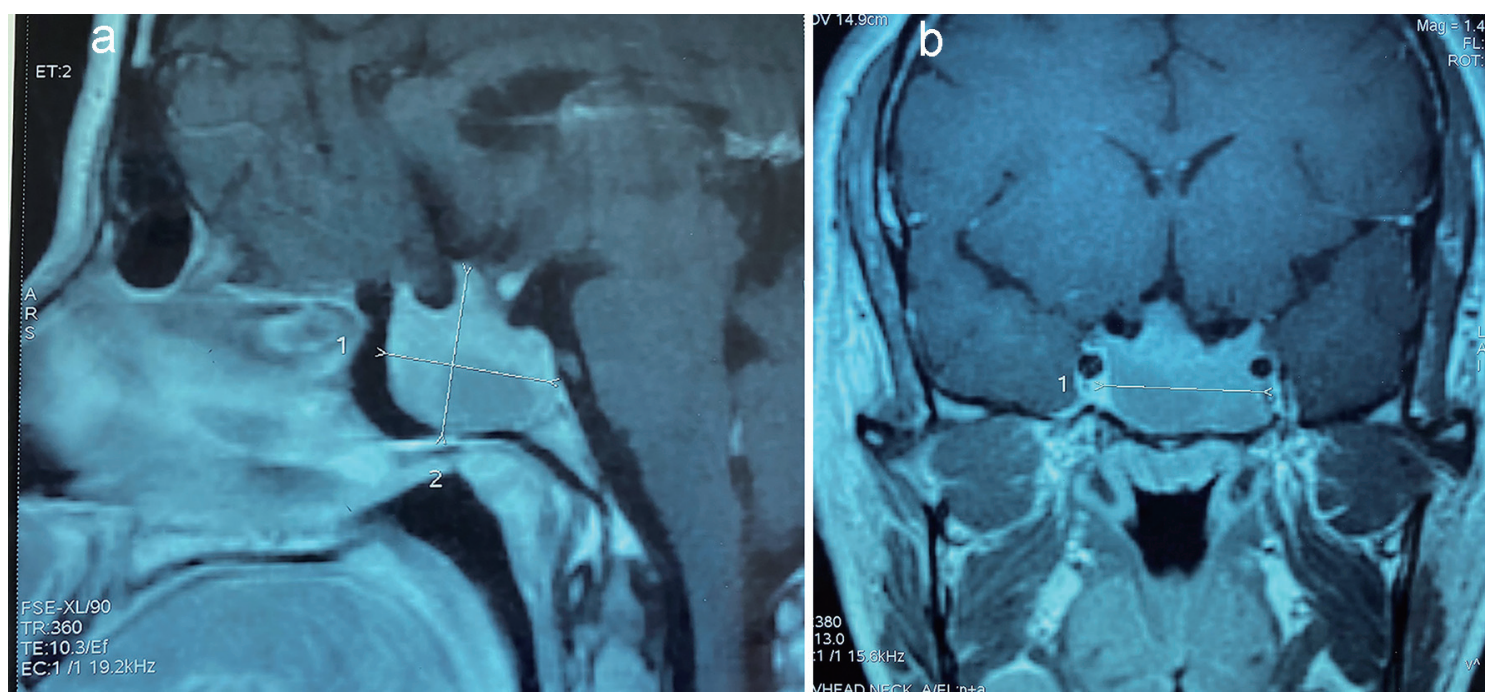

Figure 2. Sagittal (a) and coronal (b) T1-weighted magnetic resonance image showing a decrease of the adenoma size after 3 months of cabergoline treatment.

and atrophy.

The treatment of patients with macroprolactinoma complicated by optic chiasma prolapse consists of DA dosage reduction, withdrawal, or surgical treatment.

DA withdrawal and re-initiation at a lower dose is hypothesized to relieve the tension on the optic chiasm with only lim-

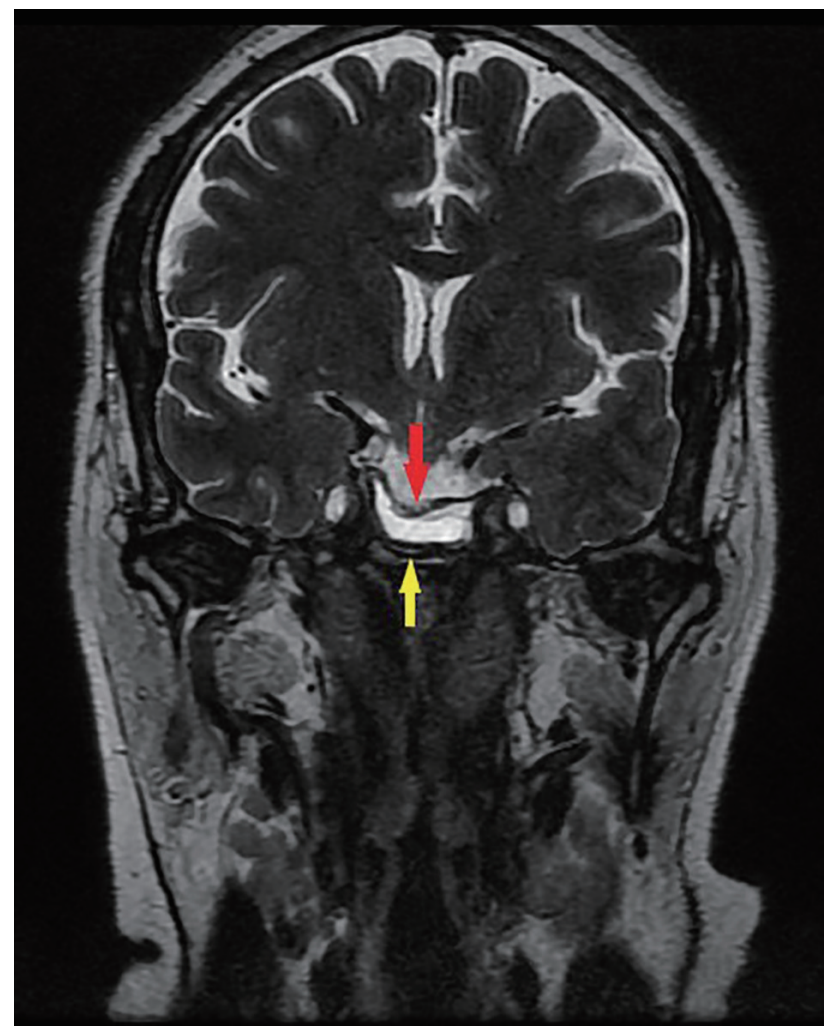

Figure 3. Coronal T2-weighted magnetic resonance image showing shrinkage of the pituitary adenoma (yellow arrow) with prolapse of optic chiasm (red arrow). ited tumor regrowth $[6,10]$. This therapeutic strategy was reported by many authors. A visual improvement to normal was reported in five cases within 2 weeks to 12 months $[6,7,9$, 10]. A significant improvement in two cases [10] and marginal improvement in one case were reported [12].

The other therapeutic alternative is surgery by performing a chiasmapexy. The goals of chiasmapexy are either lysis of the adhesions between the optic chiasm and dura or elevation of the dura itself [13]. The former requires a craniotomy and intradural exposure, and the latter can be done through an extradural minimally invasive endonasal endoscopic approach [13]. The extradural trans-sphenoidal approach reduces the risk of cerebrospinal fluid leakage as well as the risk of ischemia to the chiasm [13]. This approach was undertaken successfully in three patients with significant improvement of visual function [13-15]. However, the experience of the neurosurgeon is an important factor of success of this approach.

For our patient, we chose a medical approach because of the atrophy of optic chiasm to avoid a risk of worsening the optic chiasm impairment.

\section{Learning points}

DA remains the first choice therapy of macroprolactinomas. However, the clinician should be very careful when there is adherence between adenoma and the suprasellar visual system before treatment. In these cases, DA treatment should be initiated very gradually with regular assessment of visual field perimetry all along the treatment to avoid a dramatic shrinkage of the tumor and optic chiasm prolapse.

\section{Acknowledgments}

None to declare. 


\section{Financial Disclosure}

None to declare.

\section{Conflict of Interest}

None to declare.

\section{Informed Consent}

Written informed consent has been obtained from the patient.

\section{Author Contributions}

AM collected the case data and prepared the initial manuscript. $\mathrm{OZ}$ was involved in expert review of imaging. AM, HJ and SC proofread and revised the manuscript. CAA and GB reviewed the manuscript. All the authors have read and agreed to the final manuscript.

\section{Data Availability}

The authors declare that data supporting the findings of this study are available within the article.

\section{References}

1. Schlechte JA. Clinical practice. Prolactinoma. N Engl J Med. 2003;349(21):2035-2041.

2. Gillam MP, Molitch ME, Lombardi G, Colao A. Advances in the treatment of prolactinomas. Endocr Rev. 2006;27(5):485-534.

3. Papanastasiou L, Fountoulakis S, Pappa T, Liberopoulos K, Malliopoulos D, Markou A, Piaditis G. Brain and optic chiasmal herniation following cabergoline treatment for a giant prolactinoma: wait or intervene? Hormones (Athens). 2014;13(2):290-295.

4. Knoepfelmacher M, Gomes MC, Melo ME, Mendonca BB. Pituitary apoplexy during therapy with cabergoline in an adolescent male with prolactin-secreting macroadenoma. Pituitary. 2004;7(2):83-87.
5. Leong KS, Foy PM, Swift AC, Atkin SL, Hadden DR, MacFarlane IA. CSF rhinorrhoea following treatment with dopamine agonists for massive invasive prolactinomas. Clin Endocrinol (Oxf). 2000;52(1):43-49.

6. Jones SE, James RA, Hall K, Kendall-Taylor P. Optic chiasmal herniation - an under recognized complication of dopamine agonist therapy for macroprolactinoma. Clin Endocrinol (Oxf). 2000;53(4):529-534.

7. Taxel P, Waitzman DM, Harrington JF, Jr., Fagan RH, Rothfield NF, Chen HH, Malchoff CD. Chiasmal herniation as a complication of bromocriptine therapy. J Neuroophthalmol. 1996;16(4):252-257.

8. Wu ZB, Su ZP, Wu JS, Zheng WM, Zhuge QC, Zhong M. Five years follow-up of invasive prolactinomas with special reference to the control of cavernous sinus invasion. Pituitary. 2008;11(1):63-70.

9. Chuman H, Cornblath WT, Trobe JD, Gebarski SS. Delayed visual loss following pergolide treatment of a prolactinoma. J Neuroophthalmol. 2002;22(2):102-106.

10. Raverot G, Jacob M, Jouanneau E, Delemer B, Vighetto A, Pugeat M, Borson-Chazot F. Secondary deterioration of visual field during cabergoline treatment for macroprolactinoma. Clin Endocrinol (Oxf). 2009;70(4):588-592.

11. Kaufman B, Tomsak RL, Kaufman BA, Arafah BU, Bellon EM, Selman WR, Modic MT. Herniation of the suprasellar visual system and third ventricle into empty sellae: morphologic and clinical considerations. AJR Am J Roentgenol. 1989;152(3):597-608.

12. Dhanwal DK, Sharma AK. Brain and optic chiasmal herniations into sella after cabergoline therapy of giant prolactinoma. Pituitary. 2011;14(4):384-387.

13. Alvarez Berastegui GR, Raza SM, Anand VK, Schwartz TH. Endonasal endoscopic transsphenoidal chiasmapexy using a clival cranial base cranioplasty for visual loss from massive empty sella following macroprolactinoma treatment with bromocriptine: case report. J Neurosurg. 2016;124(4):1025-1031.

14. Kubo S, Hasegawa H, Inui T, Tominaga S, Yoshimine T. Endonasal endoscopic transsphenoidal chiasmapexy with silicone plates for empty sella syndrome: technical note. Neurol Med Chir (Tokyo). 2005;45(8):428-432; discussion 432.

15. Cobb MIH, Crowson M, Mintz-Cole R, Husain AM, Berger M, Jang D, Codd P. Transnasal transsphenoidal elevation of optic chiasm in secondary empty sella syndrome following prolactinoma treatment. World Neurosurg. 2018;112:250-253. 